\title{
Influence of Zinc Applications on Photosynthesis, Transpiration and Stomatal Conductance in Kharif Rice (Oryza sativa L.) Genotypes
}

\author{
Vivek N. Zinzala ${ }^{\text {* }}$, Ajay V. Narwade ${ }^{1}$, Nilima Karmakar ${ }^{2}$ and P. B. Patel ${ }^{3}$ \\ ${ }^{1}$ Department of Genetics and Plant Breeding, ${ }^{2}$ Department of Soil Science and Agricultural \\ Chemistry, N. M. College of Agriculture, Navsari Agricultural University, \\ Navsari, Gujarat 396450, India \\ ${ }^{3}$ Main Rice Research Center, Navsari Agricultural University, Navsari, Gujarat 396450, India \\ *Corresponding author
}

\section{A B S T R A C T}

Keywords

Rice,

Photosynthesis,

Stomatal

Conductance,

Transpiration, Zinc

Article Info

Accepted:

04 September 2019

Available Online:

10 October 2019
A field experiment in rice investigation was conducted during kharif seasons of 2017 and 2018 at college farm, Navsari Agricultural University, Navsari to study the effect of variety and zinc application on rice. The experiment was laid out in a randomized block design with factorial concept and replicated thrice. The experiment consisted of three varieties viz., IET-25450 $\left(\mathrm{V}_{1}\right)$, BPT-5204 $\left(\mathrm{V}_{2}\right)$ and IET-24766 $\left(\mathrm{V}_{3}\right)$, three soil base zinc application $\left(\mathrm{S}_{1}\right)$ control $\left(00 \mathrm{~kg} \mathrm{ZnSO}_{4} \mathrm{ha}^{-1}\right),\left(\mathrm{S}_{2}\right) 10 \mathrm{~kg} \mathrm{ZnSO} \mathrm{Zh}^{-1}$ at the time of transplanting and $\left(\mathrm{S}_{3}\right) 20 \mathrm{~kg} \mathrm{ZnSO}_{4} \mathrm{ha}^{-1}$ at the time of transplanting and two foliar $\mathrm{Zn}$ application $\left(\mathrm{F}_{1}\right) 0 \% \mathrm{Zn}$ EDTA and $\left(\mathrm{F}_{2}\right) 1 \% \mathrm{Zn}$ EDTA spray at tillering and grain filling stage. Varieties differed significantly in photosynthetic rate, stomatal conductance and transpiration rate. Among the varieties, IET-24766 gave significantly highest photosynthetic rate $\left(44.78 \mu \mathrm{Mm}^{-2} \mathrm{~s}^{-1}\right)$, stomatal conductance $\left(0.35 \mathrm{mMm}^{-2} \mathrm{~s}^{-1}\right)$ and transpiration rate $\left(5.66 \mathrm{mMm}^{-2} \mathrm{~s}^{-1}\right)$ followed by IET-25450and BPT-5204 and soil base zinc application of $20 \mathrm{~kg} \mathrm{ZnSO}_{4} \mathrm{ha}^{-1}$ at the time of transplanting as well as foliar zinc application of $1 \% \mathrm{Zn}$ EDTA spray at tillering and grain filling stage resulted in highest values in photosynthetic rate (43.52 and $43.02 \mu \mathrm{Mm}^{-2} \mathrm{~s}^{-1}$, respectively), stomatal conductance $\left(0.35\right.$ and $0.34 \mathrm{mMm}^{-2} \mathrm{~s}^{-1}$, respectively), transpiration rate (5.38 and $5.17 \mathrm{mMm}^{-2} \mathrm{~s}^{-1}$, respectively) during in pooled analysis.

\section{Introduction}

Rice (Oryza sativa L.) is the world's most important food crop and a primary source of major food staple and energy of more than half the world population, being the major source of carbohydrate and even protein. However, rice is a poor source of essential micronutrients such as Zinc (Zn). Deficiency of $\mathrm{Zn}$ in rice has been reported in lowland rice in India (Mandal, et al., 2000 and Qadar, 2002), in Brazil (Fageria, 2001; Fageria and 
Stone, 2008) and in the Philippines (De Datta, 1981). $\mathrm{Zn}$ is a vital micronutrient for plants growth and it act as cofactor for many enzymes which are involved in various physiological and biochemical functions of crop plants. There are many enzymes which require zinc as cofactor to carry their functions viz., Copper- zinc super oxide dismutase $(\mathrm{Cu} /$ Zinc SOD), peroxidase, carbonic anhydrase and catalase that plays an important role in protecting crop versus oxidative harm catalyzed by reactive oxygen species (Barak and Helmke, 1993). $\mathrm{Zn}$ is also involved with nitrogen metabolism (e.g., glutamate dehydrogenase) and anaerobic metabolism (e.g., alcohol dehydrogenase) (Fageria et al., 2011). Zn deficient plants usually have reduced leaf chlorophyll (Chl) concentration and lower $\mathrm{Chl}$ a:b ratio, which indicates damage to intrinsic quantum efficiency of the photosystem-II (PS-II) units (Chen et al., 2008). Such damage to photosynthetic centers, decreased leaf photosynthetic capacity due to a decreased number of PS-II units per unit leaf area, making them susceptible to photodamage (Chen et al., 2008 and Rehman et al., 2012).

A global study by the Food and Agriculture Organization (FAO) showed that about 30\% of the cultivated soils of the world are $\mathrm{Zn}$ deficient (Sillanpaa, 1982). Zinc deficiency in crop plants reduces not only grain yield but also the nutritional quality of the grain. However, the frequency of soil $\mathrm{Zn}$ deficiency is greater in rice than other crops, with more than $50 \%$ of the crop worldwide prone to this nutritional disorder (Hacisalihoglu and Kochian, 2003 and Rehmanet al., 2012). Therefore, $\mathrm{Zn}$ deficiency is considered as one of the most important nutritional stresses limiting rice production in Asia (Ahmad et al., 2012). Genotypic variation in rice grain $\mathrm{Zn}$ concentration might be due to the difference in physiological processes determining Zn accumulation in grains (Gaoet al., 2012). Application of $\mathrm{ZnSO}_{4}$ is the general strategy to improve grain yield and grain $\mathrm{Zn}$ concentration in cereals grown on $\mathrm{Zn}$ deficient soils. Cakmak (2008) and Rehmanet al., (2012) have reviewed several studies where $\mathrm{Zn}$ application to Zn-deficient soils significantly and remarkably increased plant growth and grain yield, grain $\mathrm{Zn}$ concentration. Most common method of $\mathrm{Zn}$ fertilization is through soil application by broadcasting, banding in vicinity of seed, or via irrigation and commonly applied in rice under lowland condition before flooding or after transplanting to prevent $\mathrm{Zn}$ deficiency and for increased grain yield (Dobermann and Fairhurst 2000, Naik and Das 2007). Zn can be absorbed by leaf stomata when applied as foliar spray and then transported via the vascular system to where it is needed (Marschner, 1995). A number of $\mathrm{Zn}$ sources $\left[\mathrm{ZnSO}_{4}, \mathrm{Zn}\left(\mathrm{NO}_{3}\right)_{2}, \mathrm{Zn}\right.$-EDTA] have been used as foliar fertilizers in a number of crops (Yoshida et al., 1970). Foliar application of $\mathrm{ZnSO}_{4}$ is effective in correcting $\mathrm{Zn}$ deficiency and improving grain $\mathrm{Zn}$ concentration (Yoshida et al., 1970, Wilhelm et al., 1988, Jiang et al., 2008, Stomph et al., 2011). Significant increases in grain yield, straw and grain $\mathrm{Zn}$ contents were observed with foliar application of $\mathrm{Zn}$ as $\mathrm{Zn}$-EDTA and $\mathrm{ZnSO}_{4}$, but the highest increase was observed with $\mathrm{Zn}$ EDTA application (Karak and Das, 2006). Although foliar application is effective in increasing seed Zn content (Welch 2002, Yang et al., 2007, Jiang et al., 2008, Cakmak, 2009), time of foliar $\mathrm{Zn}$ application is an important factor in this regard (Jiang et al., 2008; Stomph et al., 2011). Generally, large increases in grain $\mathrm{Zn}$ occur when it is foliar applied at later stages of plant development. Higher translocation of $\mathrm{Zn}$ from flag leaves to grains occurred when $\mathrm{Zn}$ had been applied at booting or anthesis stage in a nutrient solution when genotypes with high or low grain $\mathrm{Zn}$ were used (Wu et al., 2010). Foliar application of $\mathrm{Zn}$ at panicle initiation was effective in increasing whole grain $\mathrm{Zn}$ contents 2-fold 
(Phattarakul et al., 2011). The overall aim of the study is to understand the effect of soil base $\mathrm{Zn}$ application and foliar $\mathrm{Zn}$ application on effect of photosynthetic rate, stomatal conductance and transpiration rate of selected rice genotypes.

\section{Materials and Methods}

\section{Planting materials and treatments}

To study the effect of $\mathrm{Zn}$ application on physiological parameters, a field experiment was conducted during 2017 and 2018 in a randomized block design with factorial concept and replicated thrice at college farm, Navsari Agricultural University, Navsari, Gujarat, India.

The experiment consisted of three varieties viz., IET-25450 $\left(\mathrm{V}_{1}\right)$, BPT-5204 $\left(\mathrm{V}_{2}\right)$ and IET$24766\left(\mathrm{~V}_{3}\right)$, three soil base zinc application $\left(\mathrm{S}_{1}\right)$ control $\left(00 \mathrm{~kg} \mathrm{ZnSO} \mathrm{ha}^{-1}\right),\left(\mathrm{S}_{2}\right) 10 \mathrm{~kg}$ $\mathrm{ZnSO}_{4} \mathrm{ha}^{-1}$ at the time of transplanting and $\left(\mathrm{S}_{3}\right) \quad 20 \mathrm{~kg} \quad \mathrm{ZnSO}_{4} \quad \mathrm{ha}^{-1}$ at the time of transplanting followed by two foliar $\mathrm{Zn}$ application $\left(\mathrm{F}_{1}\right) 0 \% \mathrm{Zn}$ EDTA and $\left(\mathrm{F}_{2}\right) 1 \%$ Zn EDTA spray at tillering and grain filling stage. Other recommended agronomical practices in vogue were followed for reaping good crop.

The photosynthetic rate, stomatal conductance and transpiration rate were measured using portable photosynthetic meter (Model CI- 340, Handheld Photosynthetic System, CIDBioscience).

Every observation was recorded with leaf covering full window of the system of five plants in each plot at 60, 90 DAT and at harvest. It was measured in upper leaf $\left(2^{\text {nd }}\right.$ leaf from top), middle leaf ( $6^{\text {th }}$ leaf from top) and lower leaf $\left(2^{\text {nd }}\right.$ leaf from bottom). These measurements for each plot at each stage were worked out and recorded. The observations recorded during the course of investigation were tabulated and analyzed statistically to draw a valid conclusion. The data were analyzed as per the standard procedure of "Analysis of Variance" (ANOVA) as described by Gomez and Gomez (1984).

The significance of treatments was tested by ' $F$ ' test (Variance ratio). Standard error of mean (S.Em. \pm ) was computed for various factors. Critical difference (CD) was used to know the differences exist between treatment mean at 5\% level of significance where ' $F$ ' test showed significant differences among means by the following formula:

$$
\text { C.D. }=\text { S.E. }(\mathrm{d}) \times \mathrm{t}_{0.05} \text {, edf }
$$

\section{Results and Discussion}

\section{Photosynthetic rate}

\section{Effect of different variety on photosynthetic rate at $60,90 \mathrm{DAT}$ and at harvest}

An appraisal of data tabulated in Table 1, 2 and 3 represent the photosynthetic rate of rice significantly affected by different variety during both of the years and in pooled analysis.

Significantly highest mean photosynthetic rate was recorded with $\left(\mathrm{V}_{3}\right)$ IET-24766 during first and second year of experiment as well as in pooled data at 60 DAT $(44.45,45.51$ and $44.98 \mu \mathrm{Mm}^{-2} \mathrm{~s}^{-1}$, respectively), $90 \mathrm{DAT}$ (44.24, 44.83 and $44.53 \mu \mathrm{Mm}^{-2} \mathrm{~s}^{-1}$, respectively) and at harvest $(24.15,24.36$ and $24.25 \mu \mathrm{Mm}^{-2} \mathrm{~s}^{-1}$, respectively) whereas lowest mean photosynthetic rate were recorded in $\left(\mathrm{V}_{2}\right)$ BPT-5204 during year of 2017, 2018 and in pooled analysis at 60 DAT $(38.92,39.99$ and $39.46 \mu \mathrm{Mm}^{-2} \mathrm{~s}^{-1}$, respectively), $90 \mathrm{DAT}$ $\left(38.72, \quad 39.38\right.$ and $39.05 \mu \mathrm{Mm}^{-2} \mathrm{~s}^{-1}$, respectively) and at harvest (17.71, 17.97 and $17.84 \mu \mathrm{Mm}^{-2} \mathrm{~s}^{-1}$, respectively). 
Effect of soil base $\mathrm{Zn}$ application on photosynthetic rate at $60,90 \mathrm{DAT}$ and at harvest

The individual years of experimentation and in pooled analysis, the result in Table 1,2 and 3 revealed that the photosynthetic rate of rice at 60, 90 days after transplanting (DAT) and at harvest were influenced significantly due to soil base $\mathrm{Zn}$ application at the time of transplanting.

The mean photosynthetic rate showed that maximum photosynthetic rate during both the years as well as in pooled analysis at 60 DAT (42.81, 44.22 and $43.52 \mu \mathrm{Mm}^{-2} \mathrm{~s}^{-1}$, respectively), 90 DAT (42.60, 43.46 and 43.03 $\mu \mathrm{Mm}^{-2} \mathrm{~s}^{-1}$, respectively) and at harvest (21.78, 22.06 and $21.92 \mu \mathrm{Mm}^{-2} \mathrm{~s}^{-1}$, respectively)were noted under application of $20 \mathrm{~kg} \mathrm{ZnSO}_{4} \mathrm{ha}^{-}$ ${ }^{1}\left(\mathrm{~S}_{3}\right)$ but it was being at par with $\left(\mathrm{S}_{2}\right) 10 \mathrm{~kg}$ $\mathrm{ZnSO}_{4} \mathrm{ha}^{-1}$ during individual years of study and in pooled analysis at 60 DAT (41.60, 42.67 and $42.13 \mu \mathrm{Mm}^{-2} \mathrm{~s}^{-1}$, respectively), 90 DAT $\left(41.40,42.26\right.$ and $41.83 \mu \mathrm{Mm}^{-2} \mathrm{~s}^{-1}$, respectively) and at harvest $(20.75,21.00$ and 20.27 $\mu \mathrm{Mm}^{-2} \mathrm{~s}^{-1}$, respectively).

The lowest photosynthetic rate was recorded under the treatment $\left(\mathrm{S}_{1}\right) 00 \mathrm{~kg} \mathrm{ZnSO}_{4} \mathrm{ha}^{-1}$ during 20117, 2018 and in pooled analysis at 60 DAT (40.34, 41.04 and $40.69 \mu \mathrm{Mm}^{-2} \mathrm{~s}^{-1}$, respectively), 90 DAT (40.13, 40.28 and 40.20 $\mu \mathrm{Mm}^{-2} \mathrm{~s}^{-1}$, respectively) and at harvest (19.53, 19.75 and $19.64 \mu \mathrm{Mm}^{-2} \mathrm{~s}^{-1}$, respectively) except in pooled analysis at harvest.

Effect of foliar $\mathrm{Zn}$ application on photosynthetic rate at $60,90 \mathrm{DAT}$ and at harvest

It is evident from Table 1, 2 and 3 that the photosynthetic rate of rice was influenced significantly due to foliar $\mathrm{Zn}$ application at tillering stage during consecutive years of experiment and in pooled analysis
During individual years of experiment and in pooled analysis, significantly maximum mean photosynthetic rate at $60 \mathrm{DAT}(42.37,43.67$ and $43.02 \mu \mathrm{Mm}^{-2} \mathrm{~s}^{-1}$, respectively), $90 \mathrm{DAT}$ $\left(42.17, \quad 42.79\right.$ and $42.48 \mu \mathrm{Mm}^{-2} \mathrm{~s}^{-1}$, respectively) and at harvest $(21.44,21.70$ and $21.57 \mu \mathrm{Mm}^{-2} \mathrm{~s}^{-1}$, respectively) were recorded under application of $1 \% \mathrm{Zn} \operatorname{EDTA}\left(\mathrm{F}_{2}\right)$. Lowest photosynthetic rate was recorded to control $\left(\mathrm{F}_{1}\right) 0 \% \mathrm{Zn}$ EDTA during both the years of experimentation and in pooled analysis.

\section{Interaction effect on photosynthetic rate at 60, $90 \mathrm{DAT}$ and at harvest}

The interaction effects in respect of photosynthetic rate between variety and soil base $\mathrm{Zn}$ application (V $\mathrm{x} \mathrm{S}$ ), variety and foliar $\mathrm{Zn}$ application $(\mathrm{V} \quad \mathrm{x} F)$, soil base $\mathrm{Zn}$ application and foliar $\mathrm{Zn}$ application ( $\mathrm{S} \times \mathrm{F}$ ) and variety, soil base $\mathrm{Zn}$ application and foliar $\mathrm{Zn}$ application ( $\mathrm{V}$ x S x F) were did not exert any significant effect in individual years of study and in pooled analysis.

\section{Stomatal conductance}

Effect of different variety on stomatal conductance at 60, 90 DATand at harvest

Data presented in Table 4, 5 and 6 revealed that, varietal effect are significantly different for the stomatal conductance during both the years of experimentation and in pooled findings.

Variety $\left(\mathrm{V}_{3}\right)$ IET-24766 showed higher stomatal conductance during both years of experimentation and in pooled analysis at 60 DAT $\left(0.345, \quad 0.348\right.$ and $0.347 \mathrm{mMm}^{-2} \mathrm{~s}^{-1}$, respectively), 90 DAT $(0.333,0.336$ and 0.334 $\mathrm{mMm}^{-2} \mathrm{~s}^{-1}$, respectively) and at harvest $(0.135$, 0.144 and $0.139 \mathrm{mMm}^{-2} \mathrm{~s}^{-1}$, respectively), but it was remained at par with variety $\left(\mathrm{V}_{1}\right)$ IET25450 during consecutive years of experiment 
and in pooled analysis at 60 DAT (0.336, 0.349 and $0.338 \mathrm{mMm}^{-2} \mathrm{~s}^{-1}$, respectively) and 90 DAT $\left(0.324,0.327\right.$ and $0.326 \mathrm{mMm}^{-2} \mathrm{~s}^{-1}$, respectively).

Whereas, lowest mean stomatal conductance were recorded in variety $\left(\mathrm{V}_{2}\right)$ BPT-5204 during individual years of experiment and in pooled analysis at $60 \mathrm{DAT}(0.318,0.321$ and $0.319 \mathrm{mMm}^{-2} \mathrm{~s}^{-1}$, respectively), 90 DAT $(0.307$, 0.310 and $0.308 \mathrm{mMm}^{-2} \mathrm{~s}^{-1}$, respectively) and at harvest $\left(0.110,0.118\right.$ and $0.114 \mathrm{mMm}^{-2} \mathrm{~s}^{-1}$, respectively).

Effect of soil base $\mathrm{Zn}$ application on stomatal conductance at 60,90 DAT and atharvest

The result in Table 4, 5 and 6 revealed that the stomatal conductance of rice was influenced significantly due to different level of soil base $\mathrm{Zn}$ application at the time of transplanting during consecutive years of experimentation and in pooled analysis.

Maximum mean stomatal conductance was recorded during first and second year of experiment as well as in pooled data at 60 DAT $\left(0.346,0.349\right.$ and $0.348 \mathrm{mMm}^{-2} \mathrm{~s}^{-1}$, respectively), 90 DAT (0.334, 0.337 and 0.336 $\mathrm{mMm}^{-2} \mathrm{~s}^{-1}$, respectively) and at harvest $(0.137$, 0.147 and $0.142 \mathrm{mMm}^{-2} \mathrm{~s}^{-1}$, respectively)) under application of $20 \mathrm{~kg} \mathrm{ZnSO}_{4} \mathrm{ha}^{-1}\left(\mathrm{~S}_{3}\right)$.

But, application of $20 \mathrm{~kg} \mathrm{ZnSO} \mathrm{ha}^{-1}\left(\mathrm{~S}_{3}\right)$ remained at par with $\left(\mathrm{S}_{2}\right) 10 \mathrm{~kg} \mathrm{ZnSO} \mathrm{Zha}^{-1}$ during both the years as well as in pooled analysis at 60 DAT $(0.334,0.337$ and 0.335 $\mathrm{mMm}^{-2} \mathrm{~s}^{-1}$, respectively) and 90 DAT $(0.322$, 0.325 and $0.324 \mathrm{mMm}^{-2} \mathrm{~s}^{-1}$, respectively).

Application of $\left(\mathrm{S}_{1}\right) 00 \mathrm{~kg} \mathrm{ZnSO} \mathrm{ha}^{-1}$ gave lowest stomatal conductance during individual years of study and in pooled analysis at 60 DAT $\left(0.319,0.322\right.$ and $0.320 \mathrm{mMm}^{-2} \mathrm{~s}^{-1}$, respectively), 90 DAT $(0.308,0.311$ and 0.309
$\mathrm{mMm}^{-2} \mathrm{~s}^{-1}$, respectively) and at harvest $(0.110$, 0.116 and $0.113 \mathrm{mMm}^{-2} \mathrm{~s}^{-1}$, respectively).

\section{Effect of foliar $\mathbf{Z n}$ application on stomatal conductance at 60,90 DAT and at harvest}

It is evident from Table 4, 5 and 6 that the stomatal conductance of rice was influenced significantly due to foliar $\mathrm{Zn}$ application at tillering stage during individual years of experiment and in pooled finding.

During year of 2017, 2018 and in pooled analysis was recorded significantly maximum mean stomatal conductance at 60 DAT $(0.343$, 0.346 and $0.344 \mathrm{mMm}^{-2} \mathrm{~s}^{-1}$, respectively), 90 DAT $\left(0.330,0.334\right.$ and $0.332 \mathrm{mMm}^{-2} \mathrm{~s}^{-1}$, respectively) and at harvest $(0.134,0.144$ and $0.139 \mathrm{mMm}^{-2} \mathrm{~s}^{-1}$, respectively) under application of $1 \% \mathrm{Zn} \operatorname{EDTA}\left(\mathrm{F}_{2}\right)$ and lowest was recorded to control $\left(\mathrm{F}_{1}\right) 0 \%$ Zn EDTA during consecutive years of experiment and in pooled analysis at 60 DAT $(0.323,0.326$ and $0.325 \mathrm{mMm}^{-2} \mathrm{~s}^{-1}$, respectively), 90 DAT $\left(0.312, \quad 0.315\right.$ and $0.313 \mathrm{mMm}^{-2} \mathrm{~s}^{-1}$, respectively) and at harvest $(0.114,0.121$ and $0.117 \mathrm{mMm}^{-2} \mathrm{~s}^{-1}$, respectively).

\section{Interaction effect on stomatal conductance at 60,90 DAT and at harvest}

The interaction effects in respect of stomatal conductance between variety and soil base $\mathrm{Zn}$ application $(\mathrm{V} \times \mathrm{S})$, variety and foliar $\mathrm{Zn}$ application ( $\mathrm{V} \times \mathrm{F}$ ), soil base $\mathrm{Zn}$ application and foliar $\mathrm{Zn}$ application ( $\mathrm{S} \times \mathrm{F}$ ) and variety, soil base $\mathrm{Zn}$ application and foliar $\mathrm{Zn}$ application ( $\mathrm{V} \times \mathrm{S} \times \mathrm{F})$ were did not show any significant effect in both the years of study and in pooled analysis.

While at harvest during in pooled analysis, interaction effect of soil base $\mathrm{Zn}$ application and foliar $\mathrm{Zn}$ application ( $\mathrm{S} \quad \mathrm{x} \quad \mathrm{F}$ ) was significant while all other interaction were not significant. 
Treatment receiving interaction effect of ( $\mathrm{S} x$ F) soil $\mathrm{Zn}$ application and foliar $\mathrm{Zn}$ application produced significantly maximum mean stomatal conductance at harvest $(0.149$ $\left.\mathrm{mMm}^{-2} \mathrm{~s}^{-1}\right)$ was recorded under application of $20 \mathrm{~kg} \mathrm{ZnSO} \mathrm{ha}^{-1}+1 \% \mathrm{Zn} \operatorname{EDTA}\left(\mathrm{S}_{3} \mathrm{~F}_{2}\right)$ and lowest $\left(0.101 \mathrm{mMm}^{-2} \mathrm{~s}^{-1}\right)$ was recorded to control $\left(\mathrm{S}_{1} \mathrm{~F}_{1}\right) 00 \mathrm{~kg} \mathrm{ZnSO} \mathrm{haa}^{-1}+0 \% \mathrm{Zn}$ EDTA during in pooled analysis.

\section{Transpiration rate}

\section{Effect of different variety on transpiration rate at $60,90 \mathrm{DAT}$ and at harvest}

The results in Table 7,8 and 9 revealed that varieties showed significant variation on the transpiration rate during both the years of study and in pooled findings.

During year of 2017, 2018 and in pooled analysis, variety IET-24766 $\left(\mathrm{V}_{3}\right)$ showed significantly higher transpiration rate at 60 DAT $\left(5.65, \quad 5.66\right.$ and $5.66 \mathrm{mMm}^{-2} \mathrm{~s}^{-1}$, respectively), 90 DAT (5.45, 5.47 and 5.46 $\mathrm{mMm}^{-2} \mathrm{~s}^{-1}$, respectively) and at harvest (3.17, 3.36 and $3.27 \mathrm{mMm}^{-2} \mathrm{~s}^{-1}$, respectively) over rest of all other treatments.

While, significantly lowest mean transpiration rate were recorded in variety BPT-5204 $\left(\mathrm{V}_{2}\right)$ during both of the years of experiment and in pooled analysis at 60 DAT $(3.58,3.59$ and $3.58 \mathrm{mMm}^{-2} \mathrm{~s}^{-1}$, respectively), 90 DAT (3.37, 3.39 and $3.38 \mathrm{mMm}^{-2} \mathrm{~s}^{-1}$, respectively) and at harvest $\left(1.56,1.68\right.$ and $1.62 \mathrm{mMm}^{-2} \mathrm{~s}^{-1}$, respectively).

Effect of soil base $\mathrm{Zn}$ application on transpiration rate at $60,90 \mathrm{DAT}$ and at harvest

The result in Table 7, 8 and 9 revealed that the transpiration rate of rice was influenced significantly due to different level of soil base $\mathrm{Zn}$ application at the time of transplanting during both the years of experimentation and in pooled analysis. Significantly maximum mean transpiration rate during both years of experimentation and in pooled analysis at 60 DAT $\left(5.37, \quad 5.38\right.$ and $5.38 \mathrm{mMm}^{-2} \mathrm{~s}^{-1}$, respectively), 90 DAT (5.18, 5.19 and 5.19 $\mathrm{mMm}^{-2} \mathrm{~s}^{-1}$, respectively) and at harvest (2.71, 2.87 and $2.79 \mathrm{mMm}^{-2} \mathrm{~s}^{-1}$, respectively) was recorded under application of $20 \mathrm{~kg} \mathrm{ZnSO}_{4}$ ha ${ }^{1}\left(\mathrm{~S}_{3}\right)$ over rest of all other treatments.

Treatments exhibit significant superiority over control $\quad\left(\mathrm{S}_{1}\right) \quad 00 \quad \mathrm{~kg} \quad \mathrm{ZnSO}_{4} \mathrm{ha}^{-1}$ during consecutive years of experiment and in pooled analysis at 60 DAT (3.90, 3.92 and 3.91 $\mathrm{mMm}^{-2} \mathrm{~s}^{-1}$, respectively), 90 DAT $(3.69,3.71$ and $3.70 \mathrm{mMm}^{-2} \mathrm{~s}^{-1}$, respectively) and at harvest $\left(2.14,2.27\right.$ and $2.21 \mathrm{mMm}^{-2} \mathrm{~s}^{-1}$, respectively).

Effect of foliar $\mathrm{Zn}$ application on transpiration rate at $60,90 \mathrm{DAT}$ and at harvest

It is seen from the data in Table 7, 8 and 9 that the transpiration rate of rice was influenced significantly due to foliar $\mathrm{Zn}$ application at tillering stage and grain filling stage (except 60 DAT) during both the years of experimentation and in pooled analysis.

During first and second year of experiment as well as in pooled data, significantly maximum mean transpiration rate at $60 \mathrm{DAT}(5.16,5.18$ and $5.17 \mathrm{mMm}^{-2} \mathrm{~s}^{-1}$, respectively), 90 DAT (4.98, 4.99 and $4.98 \mathrm{mMm}^{-2} \mathrm{~s}^{-1}$, respectively) and at harvest $\left(2.61,2.78\right.$ and $2.69 \mathrm{mMm}^{-2} \mathrm{~s}^{-1}$, respectively) were recorded under application of $1 \% \mathrm{Zn} \operatorname{EDTA}\left(\mathrm{F}_{2}\right)$ and lowest mean transpiration rate was recorded to control $\left(\mathrm{F}_{1}\right)$ $0 \% \mathrm{Zn}$ EDTA during consecutive years of experiment and in pooled analysis at 60 DAT (4.06, 4.08 and $4.07 \mathrm{mMm}^{-2} \mathrm{~s}^{-1}$, respectively), 90 DAT $\left(3.85,3.87\right.$ and $3.86 \mathrm{mMm}^{-2} \mathrm{~s}^{-1}$, respectively) and at harvest $(2.25,2.37$ and $2.31 \mathrm{mMm}^{-2} \mathrm{~s}^{-1}$ ). 
Table.1 Photosynthetic rate $\left(\mu \mathrm{Mm}^{-2} \mathrm{~s}^{-1}\right)$ at $60 \mathrm{DAT}$

\begin{tabular}{|c|c|c|c|c|c|c|c|c|c|}
\hline & \multicolumn{3}{|c|}{$\mathbf{V}$} & \multicolumn{3}{|c|}{$\mathbf{S}$} & \multicolumn{2}{|c|}{$\mathbf{F}$} & $\mathbf{Y}$ \\
\cline { 2 - 10 } & $\mathbf{V}_{\mathbf{1}}$ & $\mathbf{V}_{\mathbf{2}}$ & $\mathbf{V}_{\mathbf{3}}$ & $\mathbf{S}_{\mathbf{1}}$ & $\mathbf{S}_{\mathbf{2}}$ & $\mathbf{S}_{\mathbf{3}}$ & $\mathbf{F}_{\mathbf{1}}$ & $\mathbf{F}_{\mathbf{2}}$ & \\
\hline $\mathbf{Y 1}$ & 41.37 & 38.92 & 44.45 & 40.34 & 41.60 & 42.81 & 40.79 & 42.37 & 41.58 \\
\hline Y2 & 42.43 & 39.99 & 45.51 & 41.04 & 42.67 & 44.22 & 41.62 & 43.67 & 42.64 \\
\hline Pooled & 41.90 & 39.46 & 44.98 & 40.69 & 42.13 & 43.52 & 41.20 & 43.02 & 42.11 \\
\hline
\end{tabular}

\begin{tabular}{|c|c|c|c|c|c|c|c|c|c|}
\hline & & $\mathbf{V}$ & $\mathbf{S}$ & $\mathbf{F}$ & V x S & $\mathbf{V} \times \mathbf{F}$ & $\mathbf{S} \times \mathbf{F}$ & $\mathbf{V} \times S \times F$ & $\mathbf{Y}$ \\
\hline \multirow[t]{2}{*}{ Y1 } & S.Em. \pm & 0.67 & 0.67 & 0.55 & 1.17 & 0.95 & 0.95 & 1.65 & \\
\hline & C.D. & 1.94 & 1.94 & 1.58 & NS & NS & NS & NS & \\
\hline \multirow[t]{2}{*}{ Y2 } & S.Em. \pm & 0.87 & 0.87 & 0.71 & 1.51 & 1.24 & 1.24 & 2.14 & \\
\hline & C.D. & 2.51 & 2.51 & 2.05 & NS & NS & NS & NS & \\
\hline \multirow[t]{2}{*}{ Pooled } & S.Em. \pm & 0.54 & 0.54 & 0.45 & 0.93 & 0.77 & 0.77 & 1.31 & 0.450 \\
\hline & C.D. & 1.54 & 1.54 & 1.26 & NS & NS & NS & NS & NS \\
\hline \multirow{2}{*}{$\begin{array}{l}\text { Interaction } \\
\text { with year }\end{array}$} & S.Em. \pm & 0.78 & 0.78 & 0.64 & 1.35 & 1.10 & 1.10 & 1.91 & \\
\hline & C.D. & NS & NS & NS & NS & NS & NS & NS & \\
\hline \multicolumn{2}{|c|}{ C.V. \% } & \multicolumn{8}{|c|}{7.86} \\
\hline
\end{tabular}

\begin{tabular}{|c|c|c|}
\hline $\mathbf{V}_{\mathbf{1}^{-}}$IET-25450 & $\mathbf{S}_{\mathbf{1}^{-}} \mathbf{0 0} \mathrm{kg} \mathrm{ZnSO}_{4} \mathbf{h a}^{-1}$ & $\mathbf{F}_{\mathbf{1}^{-}} \mathbf{0} \% \mathbf{Z n}$ EDTA \\
\hline $\mathbf{V}_{\mathbf{2}^{-}}$BPT-5204 & $\mathrm{S}_{2^{-}} 10 \mathrm{~kg} \mathrm{ZnSO}_{4} \mathrm{ha}^{-1}$ & $\mathrm{~F}_{2^{-}} \mathbf{1} \% \mathrm{Zn}$ EDTA \\
\hline $\mathbf{V}_{\mathbf{3}^{-}}$IET-24766 & $\mathrm{S}_{3^{-}}-20 \mathrm{~kg} \mathrm{ZnSO}_{4} \mathrm{ha}^{-1}$ & \\
\hline
\end{tabular}

Table.2 Photosynthetic rate $\left(\mu \mathrm{Mm}^{-2} \mathrm{~s}^{-1}\right)$ at $90 \mathrm{DAT}$

\begin{tabular}{|c|c|c|c|c|c|c|c|c|c|}
\hline & \multicolumn{3}{|c|}{$\mathbf{V}$} & \multicolumn{3}{|c|}{$\mathbf{S}$} & \multicolumn{2}{|c|}{$\mathbf{F}$} & Y \\
\cline { 2 - 10 } & $\mathbf{V}_{\mathbf{1}}$ & $\mathbf{V}_{\mathbf{2}}$ & $\mathbf{V}_{\mathbf{3}}$ & $\mathbf{S}_{\mathbf{1}}$ & $\mathbf{S}_{\mathbf{2}}$ & $\mathbf{S}_{\mathbf{3}}$ & $\mathbf{F}_{\mathbf{1}}$ & $\mathbf{F}_{\mathbf{2}}$ & \\
\hline Y1 & 41.17 & 38.72 & 44.24 & 40.13 & 41.40 & 42.60 & 40.58 & 42.17 & 41.38 \\
\hline Y2 & 41.79 & 39.38 & 44.83 & 40.28 & 42.26 & 43.46 & 41.21 & 42.79 & 42.00 \\
\hline Pooled & 41.48 & 39.05 & 44.53 & 40.20 & 41.83 & 43.03 & 40.90 & 42.48 & 41.69 \\
\hline
\end{tabular}

\begin{tabular}{|c|c|c|c|c|c|c|c|c|c|}
\hline & & $\mathbf{V}$ & $\mathbf{S}$ & $\mathbf{F}$ & $\mathrm{V} \times \mathrm{S}$ & $\mathbf{V} \times \mathbf{F}$ & $\mathbf{S} \times \mathbf{F}$ & $\operatorname{V} \times S \times F$ & $\mathbf{Y}$ \\
\hline \multirow[t]{2}{*}{ Y1 } & S.Em. \pm & 0.67 & 0.67 & 0.54 & 1.15 & 0.94 & 0.94 & 1.63 & \\
\hline & C.D. & 1.92 & 1.92 & 1.57 & NS & NS & NS & NS & \\
\hline \multirow[t]{2}{*}{ Y2 } & S.Em. \pm & 0.66 & 0.66 & 0.54 & 1.15 & 0.94 & 0.94 & 1.62 & \\
\hline & C.D. & 1.91 & 1.91 & 1.56 & NS & NS & NS & NS & \\
\hline \multirow[t]{2}{*}{ Pooled } & S.Em. \pm & 0.46 & 0.46 & 0.38 & 0.79 & 0.66 & 0.66 & 1.12 & 0.384 \\
\hline & C.D. & 1.31 & 1.31 & 1.08 & NS & NS & NS & NS & NS \\
\hline \multirow{2}{*}{$\begin{array}{c}\text { Interaction } \\
\text { with year }\end{array}$} & S.Em. \pm & 0.66 & 0.66 & 0.54 & 1.15 & 0.94 & 0.94 & 1.63 & \\
\hline & C.D. & NS & NS & NS & NS & NS & NS & NS & \\
\hline \multicolumn{2}{|c|}{ C.V. \% } & & & & & 6.77 & & & \\
\hline
\end{tabular}


Table.3 Photosynthetic rate $\left(\mu \mathrm{Mm}^{-2} \mathrm{~s}^{-1}\right)$ at harvest

\begin{tabular}{|c|c|c|c|c|c|c|c|c|c|}
\hline & \multicolumn{3}{|c|}{$\mathbf{V}$} & \multicolumn{3}{c|}{$\mathbf{S}$} & \multicolumn{2}{|c|}{$\mathbf{F}$} & $\mathbf{Y}$ \\
\cline { 2 - 11 } & $\mathbf{V}_{\mathbf{1}}$ & $\mathbf{V}_{\mathbf{2}}$ & $\mathbf{V}_{\mathbf{3}}$ & $\mathbf{S}_{\mathbf{1}}$ & $\mathbf{S}_{\mathbf{2}}$ & $\mathbf{S}_{\mathbf{3}}$ & $\mathbf{F}_{\mathbf{1}}$ & $\mathbf{F}_{\mathbf{2}}$ & \\
\hline Y1 & 20.21 & 17.71 & 24.15 & 19.53 & 20.75 & 21.78 & 19.94 & 21.44 & 20.69 \\
\hline Y2 & 20.48 & 17.97 & 24.36 & 19.75 & 21.00 & 22.06 & 20.17 & 21.70 & 20.94 \\
\hline Pooled & 20.34 & 17.84 & 24.25 & 19.64 & 20.87 & 21.92 & 20.05 & 21.57 & 20.81 \\
\hline
\end{tabular}

\begin{tabular}{|c|c|c|c|c|c|c|c|c|c|}
\hline & & V & $\mathbf{S}$ & $\mathbf{F}$ & $\mathrm{V} \times \mathrm{S}$ & $\mathbf{V} \times \mathbf{F}$ & $\mathbf{S} \times \mathbf{F}$ & $V \times S \times F$ & $\mathbf{Y}$ \\
\hline \multirow[t]{2}{*}{ Y1 } & S.Em. \pm & 0.37 & 0.37 & 0.30 & 0.65 & 0.53 & 0.53 & 0.91 & \\
\hline & C.D. & 1.07 & 1.07 & 0.88 & NS & NS & NS & NS & \\
\hline \multirow[t]{2}{*}{ Y2 } & S.Em. \pm & 0.38 & 0.38 & 0.31 & 0.66 & 0.54 & 0.54 & 0.94 & \\
\hline & C.D. & 1.10 & 1.10 & 0.90 & NS & NS & NS & NS & \\
\hline \multirow[t]{2}{*}{ Pooled } & S.Em.. \pm & 0.26 & 0.26 & 0.22 & 0.45 & 0.37 & 0.37 & 0.64 & 0.218 \\
\hline & C.D. & 0.74 & 0.74 & 0.61 & NS & NS & NS & NS & NS \\
\hline \multirow{2}{*}{$\begin{array}{c}\text { Interaction } \\
\text { with year }\end{array}$} & S.Em. \pm & 0.38 & 0.38 & 0.31 & 0.65 & 0.53 & 0.53 & 0.93 & \\
\hline & C.D. & NS & NS & NS & NS & NS & NS & NS & \\
\hline \multicolumn{2}{|c|}{ C.V. \% } & \multicolumn{8}{|c|}{7.71} \\
\hline
\end{tabular}

Table.4 Stomatal conductance $\left(\mathrm{mMm}^{-2} \mathrm{~s}^{-1}\right)$ at 60 DAT

\begin{tabular}{|c|c|c|c|c|c|c|c|c|c|}
\hline & \multicolumn{3}{|c|}{$\mathbf{V}$} & \multicolumn{3}{c|}{$\mathbf{S}$} & \multicolumn{2}{c|}{$\mathbf{F}$} & $\mathbf{Y}$ \\
\cline { 2 - 10 } & $\mathbf{V}_{\mathbf{1}}$ & $\mathbf{V}_{\mathbf{2}}$ & $\mathbf{V}_{\mathbf{3}}$ & $\mathbf{S}_{\mathbf{1}}$ & $\mathbf{S}_{\mathbf{2}}$ & $\mathbf{S}_{\mathbf{3}}$ & $\mathbf{F}_{\mathbf{1}}$ & $\mathbf{F}_{\mathbf{2}}$ & \\
\hline $\mathbf{Y 1}$ & 0.336 & 0.318 & 0.345 & 0.319 & 0.334 & 0.346 & 0.323 & 0.343 & 0.333 \\
\hline Y2 & 0.339 & 0.321 & 0.348 & 0.322 & 0.337 & 0.349 & 0.326 & 0.346 & 0.336 \\
\hline Pooled & 0.338 & 0.319 & 0.347 & 0.320 & 0.335 & 0.348 & 0.325 & 0.344 & 0.334 \\
\hline
\end{tabular}

\begin{tabular}{|c|c|c|c|c|c|c|c|c|c|}
\hline & & $\mathbf{V}$ & $\mathbf{S}$ & $\mathbf{F}$ & $\mathrm{V} \times \mathrm{S}$ & $\mathbf{V} \times \mathbf{F}$ & $S \times F$ & $V \times S \times F$ & Y \\
\hline \multirow[t]{2}{*}{ Y1 } & S.Em. \pm & 0.008 & 0.008 & 0.006 & 0.013 & 0.011 & 0.011 & 0.019 & \\
\hline & C.D. & 0.022 & 0.022 & 0.018 & NS & NS & NS & NS & \\
\hline \multirow[t]{2}{*}{ Y2 } & S.Em.. & 0.008 & 0.008 & 0.006 & 0.013 & 0.011 & 0.011 & 0.019 & \\
\hline & C.D. & 0.022 & 0.022 & 0.018 & NS & NS & NS & NS & \\
\hline \multirow[t]{2}{*}{ Pooled } & S.Em. \pm & 0.05 & 0.005 & 0.004 & 0.009 & 0.008 & 0.008 & 0.013 & 0.004 \\
\hline & C.D. & 0.015 & 0.015 & 0.012 & NS & NS & NS & NS & NS \\
\hline \multirow{2}{*}{$\begin{array}{l}\text { Interaction } \\
\text { with year }\end{array}$} & S.Em.. & 0.008 & 0.008 & 0.006 & 0.013 & 0.011 & 0.011 & 0.019 & \\
\hline & C.D. & NS & NS & NS & NS & NS & NS & NS & \\
\hline \multicolumn{2}{|c|}{ C.V. \% } & \multicolumn{8}{|c|}{9.66} \\
\hline
\end{tabular}


Table.5 Stomatal conductance $\left(\mathrm{mMm}^{-2} \mathrm{~s}^{-1}\right)$ at 90 DAT

\begin{tabular}{|c|c|c|c|c|c|c|c|c|c|}
\hline & \multicolumn{3}{|c|}{$\mathbf{V}$} & \multicolumn{3}{|c|}{$\mathbf{S}$} & \multicolumn{2}{|c|}{$\mathbf{F}$} & $\mathbf{Y}$ \\
\cline { 2 - 10 } & $\mathbf{V}_{\mathbf{1}}$ & $\mathbf{V}_{\mathbf{2}}$ & $\mathbf{V}_{\mathbf{3}}$ & $\mathbf{S}_{\mathbf{1}}$ & $\mathbf{S}_{\mathbf{2}}$ & $\mathbf{S}_{\mathbf{3}}$ & $\mathbf{F}_{\mathbf{1}}$ & $\mathbf{F}_{\mathbf{2}}$ & \\
\hline Y1 & 0.324 & 0.307 & 0.333 & 0.308 & 0.322 & 0.334 & 0.312 & 0.330 & 0.321 \\
\hline Y2 & 0.327 & 0.310 & 0.336 & 0.311 & 0.325 & 0.337 & 0.315 & 0.334 & 0.324 \\
\hline Pooled & 0.326 & 0.308 & 0.334 & 0.309 & 0.324 & 0.336 & 0.313 & 0.332 & 0.323 \\
\hline
\end{tabular}

\begin{tabular}{|c|c|c|c|c|c|c|c|c|c|}
\hline & & V & $\mathbf{S}$ & $\mathbf{F}$ & V x S & $\mathbf{V} \times \mathbf{F}$ & $S \times F$ & $V \times S \times F$ & $\mathbf{Y}$ \\
\hline \multirow{2}{*}{ Y1 } & S.Em. \pm & 0.007 & 0.007 & 0.006 & 0.013 & 0.010 & 0.010 & 0.018 & \\
\hline & C.D. & 0.021 & 0.021 & 0.017 & NS & NS & NS & NS & \\
\hline \multirow[t]{2}{*}{ Y2 } & S.Em. \pm & 0.007 & 0.007 & 0.006 & 0.012 & 0.010 & 0.010 & 0.018 & \\
\hline & C.D. & 0.021 & 0.021 & 0.017 & NS & NS & NS & NS & \\
\hline \multirow[t]{2}{*}{ Pooled } & S.Em.. & 0.005 & 0.005 & 0.004 & 0.009 & 0.007 & 0.007 & 0.012 & 0.004 \\
\hline & C.D. & 0.014 & 0.014 & 0.012 & NS & NS & NS & NS & NS \\
\hline \multirow{2}{*}{$\begin{array}{l}\text { Interaction } \\
\text { with year }\end{array}$} & S.Em. \pm & 0.007 & 0.007 & 0.006 & 0.012 & 0.010 & 0.010 & 0.018 & \\
\hline & C.D. & NS & NS & NS & NS & NS & NS & NS & \\
\hline \multicolumn{2}{|c|}{ C.V. \% } & \multicolumn{8}{|c|}{9.45} \\
\hline
\end{tabular}

Table.6 Stomatal conductance $\left(\mathrm{mMm}^{-2} \mathrm{~s}^{-1}\right)$ at harvest

\begin{tabular}{|c|c|c|c|c|c|c|c|c|c|}
\hline & \multicolumn{3}{|c|}{$\mathbf{V}$} & \multicolumn{3}{|c|}{$\mathbf{S}$} & \multicolumn{2}{|c|}{$\mathbf{F}$} & $\mathbf{Y}$ \\
\cline { 2 - 11 } & $\mathbf{V}_{\mathbf{1}}$ & $\mathbf{V}_{\mathbf{2}}$ & $\mathbf{V}_{\mathbf{3}}$ & $\mathbf{S}_{\mathbf{1}}$ & $\mathbf{S}_{\mathbf{2}}$ & $\mathbf{S}_{\mathbf{3}}$ & $\mathbf{F}_{\mathbf{1}}$ & $\mathbf{F}_{\mathbf{2}}$ & \\
\hline $\mathbf{Y 1}$ & 0.128 & 0.110 & 0.135 & 0.110 & 0.126 & 0.137 & 0.114 & 0.134 & 0.124 \\
\hline $\mathbf{Y 2}$ & 0.136 & 0.118 & 0.144 & 0.116 & 0.135 & 0.147 & 0.121 & 0.144 & 0.133 \\
\hline Pooled & 0.132 & 0.114 & 0.139 & 0.113 & 0.130 & 0.142 & 0.117 & 0.139 & 0.128 \\
\hline
\end{tabular}

\begin{tabular}{|c|c|c|c|c|c|c|}
\hline & \multicolumn{3}{|c|}{$\mathbf{F}_{\mathbf{1}}$} & \multicolumn{3}{c|}{$\mathbf{F}_{\mathbf{2}}$} \\
\cline { 2 - 7 } & $\mathbf{S}_{\mathbf{1}}$ & $\mathbf{S}_{\mathbf{2}}$ & $\mathbf{S}_{\mathbf{3}}$ & $\mathbf{S}_{\mathbf{1}}$ & $\mathbf{S}_{\mathbf{2}}$ & $\mathbf{S}_{\mathbf{3}}$ \\
\hline $\mathbf{Y 1}$ & 0.098 & 0.113 & 0.130 & 0.121 & 0.138 & 0.143 \\
\hline Y2 & 0.104 & 0.120 & 0.140 & 0.129 & 0.149 & 0.155 \\
\hline Pooled & 0.101 & 0.117 & 0.135 & 0.125 & 0.144 & 0.149 \\
\hline
\end{tabular}

\begin{tabular}{|c|c|c|c|c|c|c|c|c|c|}
\hline & & V & $\mathbf{S}$ & $\mathbf{F}$ & $V \times S$ & $V \times F$ & $\mathbf{S} \times \mathbf{F}$ & $V \times S \times F$ & $\mathbf{Y}$ \\
\hline \multirow[t]{2}{*}{ Y1 } & S.Em. \pm & 0.002 & 0.002 & 0.002 & 0.004 & 0.003 & 0.003 & 0.006 & \\
\hline & C.D. & 0.007 & 0.007 & 0.005 & NS & NS & NS & NS & \\
\hline \multirow[t]{2}{*}{ Y2 } & S.Em. \pm & 0.003 & 0.003 & 0.002 & 0.005 & 0.004 & 0.004 & 0.006 & \\
\hline & C.D. & 0.007 & 0.007 & 0.006 & NS & NS & NS & NS & \\
\hline \multirow[t]{2}{*}{ Pooled } & S.Em. \pm & 0.002 & 0.002 & 0.001 & 0.003 & 0.002 & 0.002 & 0.004 & 0.001 \\
\hline & C.D. & 0.005 & 0.005 & 0.004 & NS & NS & 0.007 & NS & 0.004 \\
\hline \multirow{2}{*}{$\begin{array}{l}\text { Interaction } \\
\text { with year }\end{array}$} & S.Em. \pm & 0.002 & 0.002 & 0.002 & 0.004 & 0.004 & 0.004 & 0.006 & \\
\hline & C.D. & NS & NS & NS & NS & NS & NS & NS & \\
\hline \multicolumn{2}{|c|}{ C.V. \% } & \multicolumn{8}{|c|}{8.08} \\
\hline
\end{tabular}


Table.7 Transpiration rate $\left(\mathrm{mMm}^{-2} \mathrm{~s}^{-1}\right)$ at 60 DAT

\begin{tabular}{|c|c|c|c|c|c|c|c|c|c|}
\hline & \multicolumn{3}{|c|}{$\mathbf{V}$} & \multicolumn{3}{|c|}{$\mathbf{S}$} & \multicolumn{2}{|c|}{$\mathbf{F}$} & $\mathbf{Y}$ \\
\cline { 2 - 11 } & $\mathbf{V}_{\mathbf{1}}$ & $\mathbf{V}_{\mathbf{2}}$ & $\mathbf{V}_{\mathbf{3}}$ & $\mathbf{S}_{\mathbf{1}}$ & $\mathbf{S}_{\mathbf{2}}$ & $\mathbf{S}_{\mathbf{3}}$ & $\mathbf{F}_{\mathbf{1}}$ & $\mathbf{F}_{\mathbf{2}}$ & \\
\hline $\mathbf{Y 1}$ & 4.61 & 3.58 & 5.65 & 3.90 & 4.56 & 5.37 & 4.06 & 5.16 & 4.61 \\
\hline Y2 & 4.63 & 3.59 & 5.66 & 3.92 & 4.59 & 5.38 & 4.08 & 5.18 & 4.63 \\
\hline Pooled & 4.62 & 3.58 & 5.66 & 3.91 & 4.58 & 5.38 & 4.07 & 5.17 & 4.62 \\
\hline
\end{tabular}

\begin{tabular}{|c|c|c|c|c|c|c|c|c|c|}
\hline & \multicolumn{3}{|c|}{$\mathbf{V}_{\mathbf{1}}$} & \multicolumn{3}{c|}{$\mathbf{V}_{\mathbf{2}}$} & \multicolumn{3}{c|}{$\mathbf{V}_{\mathbf{3}}$} \\
\cline { 2 - 10 } & $\mathbf{S}_{\mathbf{1}}$ & $\mathbf{S}_{\mathbf{2}}$ & $\mathbf{S}_{\mathbf{3}}$ & $\mathbf{S}_{\mathbf{1}}$ & $\mathbf{S}_{\mathbf{2}}$ & $\mathbf{S}_{\mathbf{3}}$ & $\mathbf{S}_{\mathbf{1}}$ & $\mathbf{S}_{\mathbf{2}}$ & $\mathbf{S}_{\mathbf{3}}$ \\
\hline $\mathbf{Y 1}$ & 3.59 & 4.72 & 5.53 & 3.00 & 3.49 & 4.24 & 5.12 & 5.49 & 6.34 \\
\hline Y2 & 3.60 & 4.76 & 5.55 & 3.02 & 3.51 & 4.26 & 5.14 & 5.50 & 6.35 \\
\hline Pooled & 3.59 & 4.74 & 5.54 & 3.01 & 3.50 & 4.25 & 5.13 & 5.49 & 6.35 \\
\hline
\end{tabular}

\begin{tabular}{|c|c|c|c|c|c|c|}
\hline & \multicolumn{2}{|c|}{$\mathbf{V}_{\mathbf{1}}$} & \multicolumn{2}{c|}{$\mathbf{V}_{\mathbf{2}}$} & \multicolumn{2}{c|}{$\mathbf{V}_{\mathbf{3}}$} \\
\hline & $\mathbf{F}_{\mathbf{1}}$ & $\mathbf{F}_{\mathbf{2}}$ & $\mathbf{F}_{\mathbf{1}}$ & $\mathbf{F}_{\mathbf{2}}$ & $\mathbf{F}_{\mathbf{1}}$ & $\mathbf{F}_{\mathbf{2}}$ \\
\hline Y1 & 3.89 & 5.33 & 3.10 & 4.05 & 5.19 & 6.11 \\
\hline Y2 & 3.91 & 5.36 & 3.12 & 4.07 & 5.20 & 6.12 \\
\hline Pooled & 3.90 & 5.35 & 3.11 & 4.06 & 5.20 & 6.12 \\
\hline
\end{tabular}

\begin{tabular}{|c|c|c|c|c|c|c|}
\hline & \multicolumn{3}{|c|}{$\mathbf{F}_{\mathbf{1}}$} & \multicolumn{3}{c|}{$\mathbf{F}_{\mathbf{2}}$} \\
\cline { 2 - 7 } & $\mathbf{S}_{\mathbf{1}}$ & $\mathbf{S}_{\mathbf{2}}$ & $\mathbf{S}_{\mathbf{3}}$ & $\mathbf{S}_{\mathbf{1}}$ & $\mathbf{S}_{\mathbf{2}}$ & $\mathbf{S}_{\mathbf{3}}$ \\
\hline $\mathbf{Y 1}$ & 3.56 & 3.86 & 4.76 & 4.24 & 5.27 & 5.98 \\
\hline Y2 & 3.57 & 3.88 & 4.77 & 4.26 & 5.29 & 6.00 \\
\hline Pooled & 3.57 & 3.87 & 4.77 & 4.25 & 5.28 & 5.99 \\
\hline
\end{tabular}

\begin{tabular}{|c|c|c|c|c|c|c|c|c|c|}
\hline & & V & $\mathbf{S}$ & $\mathbf{F}$ & $\mathbf{V} \times S$ & $\mathbf{V} \times \mathbf{F}$ & $S \times F$ & $V \times S \times F$ & $\mathbf{Y}$ \\
\hline \multirow[t]{2}{*}{ Y1 } & S.Em. \pm & 0.076 & 0.076 & 0.062 & 0.132 & 0.108 & 0.108 & 0.186 & \\
\hline & C.D. & 0.219 & 0.219 & 0.179 & 0.379 & 0.310 & 0.310 & 0.536 & \\
\hline \multirow[t]{2}{*}{ Y2 } & S.Em. \pm & 0.083 & 0.083 & 0.068 & 0.144 & 0.117 & 0.117 & 0.203 & \\
\hline & C.D. & 0.238 & 0.238 & 0.195 & 0.413 & 0.337 & 0.337 & 0.584 & \\
\hline \multirow[t]{2}{*}{ Pooled } & S.Em. \pm & 0.055 & 0.055 & 0.046 & 0.095 & 0.078 & 0.078 & 0.134 & 0.046 \\
\hline & C.D. & 0.157 & 0.157 & 0.129 & 0.267 & 0.221 & 0.221 & 0.378 & NS \\
\hline \multirow{2}{*}{$\begin{array}{c}\text { Interaction } \\
\text { with year }\end{array}$} & S.Em. \pm & 0.080 & 0.080 & 0.065 & 0.138 & 0.113 & 0.113 & 0.195 & \\
\hline & C.D. & NS & NS & NS & NS & NS & NS & NS & \\
\hline \multicolumn{2}{|c|}{ C.V. \% } & \multicolumn{8}{|c|}{7.30} \\
\hline
\end{tabular}


Table.8 Transpiration rate $\left(\mathrm{mMm}^{-2} \mathrm{~s}^{-1}\right)$ at 90 DAT

\begin{tabular}{|c|c|c|c|c|c|c|c|c|c|}
\hline & \multicolumn{3}{|c|}{$\mathbf{V}$} & \multicolumn{3}{|c|}{$\mathbf{S}$} & \multicolumn{2}{|c|}{$\mathbf{F}$} & $\mathbf{Y}$ \\
\cline { 2 - 11 } & $\mathbf{V}_{\mathbf{1}}$ & $\mathbf{V}_{\mathbf{2}}$ & $\mathbf{V}_{\mathbf{3}}$ & $\mathbf{S}_{\mathbf{1}}$ & $\mathbf{S}_{\mathbf{2}}$ & $\mathbf{S}_{\mathbf{3}}$ & $\mathbf{F}_{\mathbf{1}}$ & $\mathbf{F}_{\mathbf{2}}$ & \\
\hline $\mathbf{Y 1}$ & 4.42 & 3.37 & 5.45 & 3.69 & 4.38 & 5.18 & 3.85 & 4.98 & 4.42 \\
\hline Y2 & 4.43 & 3.39 & 5.47 & 3.71 & 4.39 & 5.19 & 3.87 & 4.99 & 4.43 \\
\hline Pooled & 4.43 & 3.38 & 5.46 & 3.70 & 4.38 & 5.19 & 3.86 & 4.98 & 4.42 \\
\hline
\end{tabular}

\begin{tabular}{|c|c|c|c|c|c|c|c|c|c|}
\hline & \multicolumn{3}{|c|}{$\mathbf{V}_{\mathbf{1}}$} & \multicolumn{3}{c|}{$\mathbf{V}_{\mathbf{2}}$} & \multicolumn{3}{c|}{$\mathbf{V}_{\mathbf{3}}$} \\
\cline { 2 - 10 } & $\mathbf{S}_{\mathbf{1}}$ & $\mathbf{S}_{\mathbf{2}}$ & $\mathbf{S}_{\mathbf{3}}$ & $\mathbf{S}_{\mathbf{1}}$ & $\mathbf{S}_{\mathbf{2}}$ & $\mathbf{S}_{\mathbf{3}}$ & $\mathbf{S}_{\mathbf{1}}$ & $\mathbf{S}_{\mathbf{2}}$ & $\mathbf{S}_{\mathbf{3}}$ \\
\hline Y1 & 3.35 & 4.56 & 5.35 & 2.82 & 3.29 & 4.02 & 4.91 & 5.29 & 6.17 \\
\hline Y2 & 3.37 & 4.57 & 5.36 & 2.83 & 3.30 & 4.03 & 4.92 & 5.30 & 6.19 \\
\hline Pooled & 3.36 & 4.56 & 5.35 & 2.82 & 3.29 & 4.03 & 4.91 & 5.29 & 6.18 \\
\hline
\end{tabular}

\begin{tabular}{|c|c|c|c|c|c|c|}
\hline & \multicolumn{2}{|c|}{$\mathbf{V}_{\mathbf{1}}$} & \multicolumn{2}{c|}{$\mathbf{V}_{\mathbf{2}}$} & \multicolumn{2}{c|}{$\mathbf{V}_{\mathbf{3}}$} \\
\hline & $\mathbf{F}_{\mathbf{1}}$ & $\mathbf{F}_{\mathbf{2}}$ & $\mathbf{F}_{\mathbf{1}}$ & $\mathbf{F}_{\mathbf{2}}$ & $\mathbf{F}_{\mathbf{1}}$ & $\mathbf{F}_{\mathbf{2}}$ \\
\hline Y1 & 3.70 & 5.14 & 2.88 & 3.87 & 4.98 & 5.93 \\
\hline Y2 & 3.71 & 5.15 & 2.90 & 3.88 & 5.00 & 5.93 \\
\hline Pooled & 3.70 & 5.15 & 2.89 & 3.87 & 4.99 & 5.93 \\
\hline
\end{tabular}

\begin{tabular}{|c|c|c|c|c|c|c|}
\hline \multirow{2}{*}{} & \multicolumn{3}{|c|}{$\mathbf{F}_{\mathbf{1}}$} & \multicolumn{3}{c|}{$\mathbf{F}_{\mathbf{2}}$} \\
\cline { 2 - 7 } & $\mathbf{S}_{\mathbf{1}}$ & $\mathbf{S}_{\mathbf{2}}$ & $\mathbf{S}_{\mathbf{3}}$ & $\mathbf{S}_{\mathbf{1}}$ & $\mathbf{S}_{\mathbf{2}}$ & $\mathbf{S}_{\mathbf{3}}$ \\
\hline Y1 & 3.34 & 3.65 & 4.57 & 4.05 & 5.10 & 5.79 \\
\hline Y2 & 3.35 & 3.67 & 4.59 & 4.06 & 5.11 & 5.80 \\
\hline Pooled & 3.34 & 3.66 & 4.58 & 4.05 & 5.10 & 5.79 \\
\hline
\end{tabular}

\begin{tabular}{|c|c|c|c|c|c|c|c|c|c|}
\hline & & V & $\mathbf{S}$ & $\mathbf{F}$ & $V \times S$ & V x F & S XF & $V \times S \times F$ & $\mathbf{Y}$ \\
\hline \multirow[t]{2}{*}{ Y1 } & S.Em. \pm & 0.08 & 0.08 & 0.06 & 0.13 & 0.11 & 0.11 & 0.19 & \\
\hline & C.D. & 0.22 & 0.22 & 0.18 & 0.38 & 0.31 & 0.31 & 0.54 & \\
\hline \multirow[t]{2}{*}{ Y2 } & S.Em. \pm & 0.08 & 0.08 & 0.06 & 0.13 & 0.11 & 0.11 & 0.19 & \\
\hline & C.D. & 0.22 & 0.22 & 0.18 & 0.38 & 0.31 & 0.31 & 0.54 & \\
\hline \multirow[t]{2}{*}{ Pooled } & S.Em. \pm & 0.05 & 0.05 & 0.04 & 0.09 & 0.08 & 0.08 & 0.13 & 0.044 \\
\hline & C.D. & 0.15 & 0.15 & 0.12 & 0.26 & 0.21 & 0.21 & 0.36 & NS \\
\hline \multirow{2}{*}{$\begin{array}{c}\text { Interaction } \\
\text { with year }\end{array}$} & S.Em. \pm & 0.08 & 0.08 & 0.06 & 0.13 & 0.11 & 0.11 & 0.19 & \\
\hline & C.D. & NS & NS & NS & NS & NS & NS & NS & \\
\hline \multicolumn{2}{|c|}{ C.V. \% } & \multicolumn{8}{|c|}{7.32} \\
\hline
\end{tabular}


Int.J.Curr.Microbiol.App.Sci (2019) 8(10): 150-168

Table.9 Transpiration rate $\left(\mathrm{mMm}^{-2} \mathrm{~s}^{-1}\right)$ at harvest

\begin{tabular}{|c|c|c|c|c|c|c|c|c|c|}
\hline & \multicolumn{3}{|c|}{$\mathbf{V}$} & \multicolumn{3}{|c|}{$\mathbf{S}$} & \multicolumn{2}{|c|}{$\mathbf{F}$} & $\mathbf{Y}$ \\
\cline { 2 - 11 } & $\mathbf{V}_{\mathbf{1}}$ & $\mathbf{V}_{\mathbf{2}}$ & $\mathbf{V}_{\mathbf{3}}$ & $\mathbf{S}_{\mathbf{1}}$ & $\mathbf{S}_{\mathbf{2}}$ & $\mathbf{S}_{\mathbf{3}}$ & $\mathbf{F}_{\mathbf{1}}$ & $\mathbf{F}_{\mathbf{2}}$ & \\
\hline $\mathbf{Y 1}$ & 2.55 & 1.56 & 3.17 & 2.14 & 2.43 & 2.71 & 2.25 & 2.61 & 2.43 \\
\hline Y2 & 2.69 & 1.68 & 3.36 & 2.27 & 2.58 & 2.87 & 2.37 & 2.78 & 2.58 \\
\hline Pooled & 2.62 & 1.62 & 3.27 & 2.21 & 2.51 & 2.79 & 2.31 & 2.69 & 2.50 \\
\hline
\end{tabular}

\begin{tabular}{|c|c|c|c|c|c|c|c|c|c|}
\hline & \multicolumn{3}{|c|}{$\mathbf{V}_{\mathbf{1}}$} & \multicolumn{3}{c|}{$\mathbf{V}_{\mathbf{2}}$} & \multicolumn{3}{c|}{$\mathbf{V}_{\mathbf{3}}$} \\
\cline { 2 - 10 } & $\mathbf{S}_{\mathbf{1}}$ & $\mathbf{S}_{\mathbf{2}}$ & $\mathbf{S}_{\mathbf{3}}$ & $\mathbf{S}_{\mathbf{1}}$ & $\mathbf{S}_{\mathbf{2}}$ & $\mathbf{S}_{\mathbf{3}}$ & $\mathbf{S}_{\mathbf{1}}$ & $\mathbf{S}_{\mathbf{2}}$ & $\mathbf{S}_{\mathbf{3}}$ \\
\hline $\mathbf{Y 1}$ & 2.05 & 2.56 & 3.05 & 1.43 & 1.57 & 1.68 & 2.95 & 3.17 & 3.40 \\
\hline Y2 & 2.17 & 2.68 & 3.21 & 1.52 & 1.70 & 1.82 & 3.13 & 3.35 & 3.59 \\
\hline Pooled & 2.11 & 2.62 & 3.13 & 1.48 & 1.64 & 1.75 & 3.04 & 3.26 & 3.50 \\
\hline
\end{tabular}

\begin{tabular}{|c|c|c|c|c|c|c|}
\hline \multirow{2}{*}{} & \multicolumn{2}{|c|}{$\mathbf{V}_{\mathbf{1}}$} & \multicolumn{2}{c|}{$\mathbf{V}_{\mathbf{2}}$} & \multicolumn{2}{c|}{$\mathbf{V}_{\mathbf{3}}$} \\
\cline { 2 - 7 } & $\mathbf{F}_{\mathbf{1}}$ & $\mathbf{F}_{\mathbf{2}}$ & $\mathbf{F}_{\mathbf{1}}$ & $\mathbf{F}_{\mathbf{2}}$ & $\mathbf{F}_{\mathbf{1}}$ & $\mathbf{F}_{\mathbf{2}}$ \\
\hline Y1 & 2.27 & 2.84 & 1.46 & 1.66 & 3.03 & 3.32 \\
\hline Y2 & 2.35 & 3.02 & 1.57 & 1.80 & 3.20 & 3.52 \\
\hline Pooled & 2.31 & 2.93 & 1.51 & 1.73 & 3.11 & 3.42 \\
\hline
\end{tabular}

\begin{tabular}{|c|c|c|c|c|c|c|}
\hline & \multicolumn{3}{|c|}{$\mathbf{F}_{\mathbf{1}}$} & \multicolumn{3}{c|}{$\mathbf{F}_{\mathbf{2}}$} \\
\cline { 2 - 7 } & $\mathbf{S}_{\mathbf{1}}$ & $\mathbf{S}_{\mathbf{2}}$ & $\mathbf{S}_{\mathbf{3}}$ & $\mathbf{S}_{\mathbf{1}}$ & $\mathbf{S}_{\mathbf{2}}$ & $\mathbf{S}_{\mathbf{3}}$ \\
\hline Y1 & 1.99 & 2.15 & 2.61 & 2.29 & 2.71 & 2.81 \\
\hline Y2 & 2.08 & 2.28 & 2.76 & 2.46 & 2.88 & 2.99 \\
\hline Pooled & 2.04 & 2.22 & 2.68 & 2.38 & 2.80 & 2.90 \\
\hline
\end{tabular}

\begin{tabular}{|c|c|c|c|c|c|c|c|c|c|}
\hline & & V & $\mathbf{S}$ & F & $\mathbf{V} \times S$ & V x F & S x F & $\mathrm{V} \times S \times F$ & $\mathbf{Y}$ \\
\hline \multirow[t]{2}{*}{ Y1 } & S.Em. \pm & 0.035 & 0.035 & 0.029 & 0.061 & 0.050 & 0.050 & 0.087 & \\
\hline & C.D. & 0.102 & 0.102 & 0.083 & 0.177 & 0.144 & 0.144 & 0.250 & \\
\hline \multirow[t]{2}{*}{ Y2 } & S.Em. \pm & 0.039 & 0.039 & 0.032 & 0.068 & 0.056 & 0.056 & 0.097 & \\
\hline & C.D. & 0.113 & 0.113 & 0.093 & 0.196 & 0.160 & 0.160 & 0.278 & \\
\hline \multirow[t]{2}{*}{ Pooled } & S.Em. \pm & 0.026 & 0.026 & 0.022 & 0.045 & 0.037 & 0.037 & 0.063 & 0.022 \\
\hline & C.D. & 0.074 & 0.074 & 0.061 & 0.126 & 0.105 & 0.104 & 0.178 & 0.061 \\
\hline \multirow{2}{*}{$\begin{array}{c}\text { Interaction } \\
\text { with year }\end{array}$} & S.Em. \pm & 0.038 & 0.038 & 0.031 & 0.065 & 0.053 & 0.053 & 0.092 & \\
\hline & C.D. & NS & NS & NS & NS & NS & NS & NS & \\
\hline \multicolumn{2}{|c|}{ C.V. \% } & \multicolumn{8}{|c|}{6.36} \\
\hline
\end{tabular}


Table.10 Interaction effect

\begin{tabular}{|c|c|c|c|c|c|c|c|c|c|}
\hline \multicolumn{4}{|c|}{60 DAT } & \multicolumn{3}{|c|}{90 DAT } & \multicolumn{3}{|c|}{ At harvest } \\
\hline Treatments & $\begin{array}{c}\text { Year } \\
1\end{array}$ & $\begin{array}{c}\text { Year } \\
2\end{array}$ & $\begin{array}{c}\text { Year } \\
\text { pooled }\end{array}$ & $\begin{array}{c}\text { Year } \\
1\end{array}$ & $\begin{array}{c}\text { Year } \\
2\end{array}$ & $\begin{array}{c}\text { Year } \\
\text { pooled }\end{array}$ & $\begin{array}{c}\text { Year } \\
1\end{array}$ & $\begin{array}{c}\text { Year } \\
2\end{array}$ & $\begin{array}{c}\text { Year } \\
\text { pooled }\end{array}$ \\
\hline $\mathbf{V}_{\mathbf{1}} \mathbf{S}_{\mathbf{1}} \mathbf{F}_{\mathbf{1}}$ & 3.18 & 3.19 & 3.18 & 2.94 & 2.96 & 2.95 & 1.86 & 1.92 & 1.89 \\
\hline $\mathbf{V}_{1} \mathbf{S}_{1} \mathbf{F}_{2}$ & 4.00 & 4.01 & 4.00 & 3.77 & 3.78 & 3.77 & 2.23 & 2.42 & 2.33 \\
\hline $\mathbf{V}_{1} \mathbf{S}_{2} \mathbf{F}_{1}$ & 3.60 & 3.61 & 3.61 & 3.40 & 3.41 & 3.40 & 2.04 & 2.10 & 2.07 \\
\hline $\mathbf{V}_{1} S_{2} F_{2}$ & 5.84 & 5.91 & 5.87 & 5.72 & 5.73 & 5.72 & 3.08 & 3.27 & 3.17 \\
\hline $\mathbf{V}_{\mathbf{1}} \mathbf{S}_{\mathbf{3}} \mathbf{F}_{\mathbf{1}}$ & 4.90 & 4.92 & 4.91 & 4.75 & 4.77 & 4.76 & 2.89 & 3.04 & 2.97 \\
\hline $\mathbf{V}_{1} \mathbf{S}_{3} \mathbf{F}_{2}$ & 6.16 & 6.18 & 6.17 & 5.94 & 5.95 & 5.94 & 3.21 & 3.37 & 3.29 \\
\hline $\mathbf{V}_{2} \mathbf{S}_{1} \mathbf{F}_{1}$ & 2.79 & 2.81 & 2.80 & 2.57 & 2.58 & 2.58 & 1.34 & 1.42 & 1.38 \\
\hline $\mathbf{V}_{2} S_{1} F_{2}$ & 3.21 & 3.22 & 3.21 & 3.06 & 3.07 & 3.07 & 1.52 & 1.63 & 1.57 \\
\hline $\mathbf{V}_{2} \mathbf{S}_{2} \mathbf{F}_{1}$ & 2.98 & 3.02 & 3.00 & 2.78 & 2.80 & 2.79 & 1.43 & 1.55 & 1.49 \\
\hline $\mathrm{V}_{2} \mathrm{~S}_{2} \mathrm{~F}_{2}$ & 3.99 & 4.00 & 3.99 & 3.79 & 3.80 & 3.79 & 1.71 & 1.86 & 1.79 \\
\hline $\mathrm{V}_{2} \mathrm{~S}_{3} \mathrm{~F}_{1}$ & 3.52 & 3.53 & 3.52 & 3.29 & 3.31 & 3.30 & 1.61 & 1.73 & 1.67 \\
\hline $\mathbf{V}_{2} \mathbf{S}_{3} \mathbf{F}_{2}$ & 4.95 & 4.98 & 4.97 & 4.75 & 4.76 & 4.75 & 1.75 & 1.90 & 1.83 \\
\hline $\mathbf{V}_{\mathbf{3}} \mathbf{S}_{\mathbf{1}} \mathbf{F}_{\mathbf{1}}$ & 4.71 & 4.72 & 4.71 & 4.50 & 4.52 & 4.51 & 2.77 & 2.91 & 2.84 \\
\hline $\mathbf{V}_{3} S_{1} F_{2}$ & 5.52 & 5.56 & 5.54 & 5.31 & 5.32 & 5.31 & 3.13 & 3.34 & 3.23 \\
\hline $\mathbf{V}_{3} \mathbf{S}_{2} \mathbf{F}_{1}$ & 5.00 & 5.03 & 5.01 & 4.78 & 4.80 & 4.79 & 2.99 & 3.18 & 3.09 \\
\hline $\mathbf{V}_{3} \mathbf{S}_{2} \mathbf{F}_{2}$ & 5.97 & 5.98 & 5.97 & 5.79 & 5.80 & 5.79 & 3.35 & 3.53 & 3.44 \\
\hline $\mathbf{V}_{3} \mathbf{S}_{3} \mathbf{F}_{1}$ & 5.85 & 5.87 & 5.86 & 5.66 & 5.68 & 5.67 & 3.33 & 3.50 & 3.41 \\
\hline $\mathbf{V}_{3} S_{3} F_{2}$ & 6.83 & 6.84 & 6.83 & 6.68 & 6.69 & 6.68 & 3.47 & 3.69 & 3.58 \\
\hline S.Em. \pm & 0.19 & 0.203 & 0.03 & 0.19 & 0.19 & 0.13 & 0.09 & 0.10 & 0.06 \\
\hline C.D.@ 5 \% & 0.54 & 0.584 & 0.38 & 0.54 & 0.54 & 0.36 & 0.25 & 0.28 & 0.18 \\
\hline
\end{tabular}

Interaction effect on transpiration rate at 60, 90 DAT and at harvest

The interaction effects of transpiration rate (Table 10) between variety and soil base $\mathrm{Zn}$ application ( $\mathrm{V} \times \mathrm{S}$ ), variety and foliar $\mathrm{Zn}$ application ( $\mathrm{V} \times \mathrm{F}$ ), soil base $\mathrm{Zn}$ application and foliar $\mathrm{Zn}$ application ( $\mathrm{S} \times \mathrm{F}$ ) and variety, soil base $\mathrm{Zn}$ application and foliar $\mathrm{Zn}$ application ( $\mathrm{V} \times \mathrm{S} \times \mathrm{F}$ ) shows significantly in individual years of study and in pooled analysis.

The interaction effects (V x S) during 2017, 2018 and in pooled analysis were recorded significantly higher transpiration rate at 60 DAT $\left(6.34,6.35\right.$ and $6.35 \mathrm{mMm}^{-2} \mathrm{~s}^{-1}$, respectively), 90 DAT $(6.17,6.19$ and 6.18 $\mathrm{mMm}^{-2} \mathrm{~s}^{-1}$, respectively) and at harvest (3.40,
3.59 and $3.50 \mathrm{mMm}^{-2} \mathrm{~s}^{-1}$, respectively) in the interaction $\mathrm{V}_{3} \mathrm{~S}_{3}$ (IET-24766 + $20 \mathrm{~kg} \mathrm{ZnSO}_{4}$ $\mathrm{ha}^{-1}$ ) and the lowest mean transpiration rate at 60 DAT (3.00, 3.02 and $\left.3.01 \mathrm{mMm}^{-2} \mathrm{~s}^{-1}\right), 90$ DAT $\left(2.82,2.83\right.$ and $2.82 \mathrm{mMm}^{-2} \mathrm{~s}^{-1}$, respectively) and at harvest $(1.43,1.52$ and $1.48 \mathrm{mMm}^{-2} \mathrm{~s}^{-1}$, respectively) were recorded in the treatment combination $\mathrm{V}_{2} \mathrm{~S}_{1}$ (BPT-5204+ $00 \mathrm{~kg} \quad \mathrm{ZnSO}_{4} \mathrm{ha}^{-1}$ ) during both years of experimentation and in pooled data, respectively.

The interaction effects of variety and foliar application ( $\mathrm{V} \times \mathrm{F}$ ) during consecutive years of experiment and in pooled analysis were recorded significantly maximum mean transpiration rate at $60 \mathrm{DAT}(6.11,6.12$ and $6.12 \mathrm{mMm}^{-2} \mathrm{~s}^{-1}$, respectively), 90 DAT (5.93, 5.93 and $5.93 \mathrm{mMm}^{-2} \mathrm{~s}^{-1}$, respectively) and at 
harvest (3.32, 3.52 and $3.42 \mathrm{mMm}^{-2} \mathrm{~s}^{-1}$, respectively) under interaction $\mathrm{V}_{3} \mathrm{~F}_{2}$ (IET$24766+1.0 \%$ Zn EDTA). Whereas, the lowest transpiration rate were recorded in the treatment combination $\mathrm{V}_{2} \mathrm{~F}_{1}$ (BPT-5204 +0.0 $\% \quad \mathrm{Zn}$ EDTA) during both year of experimentation and in pooled analysis at 60 DAT $\left(3.10, \quad 3.12\right.$ and $3.11 \mathrm{mMm}^{-2} \mathrm{~s}^{-1}$, respectively), 90 DAT $(2.88,2.90$ and 2.89 $\mathrm{mMm}^{-2} \mathrm{~s}^{-1}$, respectively) and at harvest (1.46, 1.57 and $1.51 \mathrm{mMm}^{-2} \mathrm{~s}^{-1}$, respectively).

Treatment receiving interaction effect of $(\mathrm{S} x$ F) soil $\mathrm{Zn}$ application $\left(20 \mathrm{~kg} \mathrm{ZnSO}_{4} \mathrm{ha}^{-1}\right)$ and foliar $\mathrm{Zn}$ application (1.0\% Zn EDTA) $\left(\mathrm{S}_{3} \mathrm{~F}_{2}\right)$ produced significantly higher transpiration rate during individual years of experiment and in pooled analysisat 60 DAT $(5.98,6.00$ and $5.99 \mathrm{mMm}^{-2} \mathrm{~s}^{-1}$,respectively), 90 DAT (5.79, 5.80 and $5.79 \mathrm{mMm}^{-2} \mathrm{~s}^{-1}$, respectively) and at harvest $\left(2.81,2.99\right.$ and $2.90 \mathrm{mMm}^{-2} \mathrm{~s}^{-1}$, respectively). While, lowest mean transpiration rate were recorded in $\mathrm{S}_{1} \mathrm{~F}_{1}$ Control (00 kg ZnSO $\mathrm{ha}^{-1}+0.0 \% \mathrm{Zn}$ EDTA) in both years of experiment and in pooled data at 60 DAT $\left(3.56,3.57\right.$ and $3.57 \mathrm{mMm}^{-2} \mathrm{~s}^{-1}$, respectively), 90 DAT $(3.34,3.35$ and 3.34 $\mathrm{mMm}^{-2} \mathrm{~s}^{-1}$, respectively) and at harvest (1.99, 2.08 and $2.04 \mathrm{mMm}^{-2} \mathrm{~s}^{-1}$, respectively).

During consecutive years of experiment as well as in pooled analysis, the interaction effects ( $\left.\begin{array}{lllllll}V & x & S & x & F\end{array}\right)$ were recorded significantly. The data revealed the highest mean transpiration rate at 60 DAT $(6.83,6.84$ and $6.83 \mathrm{mMm}^{-2} \mathrm{~s}^{-1}$, respectively), 90 DAT (6.68, 6.69 and $6.68 \mathrm{mMm}^{-2} \mathrm{~s}^{-1}$, respectively) and at harvest $\left(3.47,3.69\right.$ and $3.58 \mathrm{mMm}^{-2} \mathrm{~s}^{-1}$, respectively) was recorded significantly in the treatment combination $\mathrm{V}_{3} \mathrm{~S}_{3} \mathrm{~F}_{2}$ (IET-24766 + $20 \mathrm{~kg} \mathrm{ZnSO}_{4} \mathrm{ha}^{-1}+1 \% \mathrm{Zn}$ EDTA). Whereas, lowest mean transpiration rate at 60 DAT $\left(2.79,2.81\right.$ and $2.80 \mathrm{mMm}^{-2} \mathrm{~s}^{-1}$, respectively), 90 DAT $\left(2.57,2.58\right.$ and $2.58 \mathrm{mMm}^{-2} \mathrm{~s}^{-1}$, respectively) and at harvest $(1.34,1.42$ and $1.38 \mathrm{mMm}^{-2} \mathrm{~s}^{-1}$, respectively) were recorded in the treatment combination $\mathrm{V}_{2} \mathrm{~S}_{1} \mathrm{~F}_{1}$ (BPT-5204 + $00 \mathrm{~kg} \mathrm{ZnSO}_{4} \mathrm{ha}^{-1}+0 \% \mathrm{Zn}$ EDTA).

The photosynthetic rate, stomatal conductance and transpiration rate were highest at 60 DAT thereafter decreases. Significantly maximum photosynthetic rate were recorded with IET24766 over IET-25450 and BPT-5204. The changes in the photosynthesis in the cultivars was due to changes in rubisco activity, protein content and chlorophyll per unit leaf area could have been caused by an alteration of rice leaf thickness or photosynthetic components per unit cell or per unit chloroplast. There was variation in the stomatal conductance might be due to genetic variation to having highest number of stomata. These findings are strongly supported by Dore et al., (2018).

The application of soil base $\mathrm{Zn}$ application (20 $\mathrm{kg} \mathrm{ZnSO}_{4} \mathrm{ha}^{-1}$ ) and foliar $\mathrm{Zn}$ application (1\% $\mathrm{Zn}$ EDTA) were recorded significantly higher photosynthetic rate, stomatal conductance and transpiration rate over rest of other treatment. Zn deficiency depressed plant leaf photosynthetic capacity may be associated to decrease in intercellular $\mathrm{CO}_{2}$ concentration and function of stomata (Sharma et al., 1994). Stomatal closure allows plants to limit transpiration, but it also limits $\mathrm{CO}_{2}$ absorption, which leads to a decreased photosynthetic activity (Sharma et al., 1995). Reported a significant influence of Zinc in the regulation of the stomatal aperture, which is accounted for possible role of $\mathrm{Zn}$ in maintaining a high $\mathrm{K}$ content in guard cells. A decrease in carbonic anhydrase activity due to $\mathrm{Zn}$ deficiency may also contribute to the reduced net photosynthetic rate (Hacisalihoglu et al., 2003). In addition, the accumulation of saccharides in leaves may be an important factor for the reducing photosynthesis activity under Zn-deficiency (Cakmak, 2000 and Dore et al., 2018). Zinc is a constituent of carbonic anhydrase and is required for the activity of ribulose 1, 5-bisphosphate carboxylase/ 
oxygenase (Rubisco) (Shrivastava and Gupta, 1996 and Storey, 2007), the photosynthetic enzymes catalyzing the diffusion of $\mathrm{CO}_{2}$ through the cell to the chloroplasts (Hatch and Slack, 1970). Zn-deficient plants usually have reduced leaf chlorophyll (Chl) concentration and lower $\mathrm{Chl}$ a:b ratio, which indicates damage to intrinsic quantum efficiency of the photosystem-II (PSII) units (Chen et al., 2008). It can be attributed to reduced antioxidant enzyme activities and high oxidative stress damage in chloroplasts due to a blockage of energy spillover from PS-II to photosystem-I (PS-I) (Chen et al., 2009). Such damage to photosynthetic centers, decreased leaf photosynthetic capacity due to a decreased number of PS-II units per unit leaf area, making them susceptible to photodamage (Chen 2008 and Rehman et al., 2012). These findings are in agreement with those obtained by Dore et al., (2018).

The causes of photoinhibition could be lower $\mathrm{Chl}$ content in $\mathrm{Zn}$-deficient leaves. Leaf Chl concentration has a crucial role for the susceptibility to photoinhibition and leaves with less $\mathrm{Chl}$ are more susceptible to photoinhibition (Pätsikkäet al., 2002). Moreover, $\mathrm{Zn}$ deficiency conditions likely cause an excess of reducing power (NADP + $\mathrm{H}^{+}$) because of reduction in the $\mathrm{CO}_{2}$ available at carboxylation sites i.e. stomatal closure (Harbinson 1994). In addition, simultaneous reduction of $\mathrm{qP}$ and $\mathrm{qN}$ indicates an over excitation of the photochemical system likely accompanied by accumulation of reduced electron acceptors.

Under such conditions the probability of generation of reactive radicals which further injure PSII components is very high (Barber and Anderson 1992). With more severe Zn deficiency or longer growth period under deficiency conditions serious damage to photosystems was expected to occur in $\mathrm{Zn}$ deficient leaves.
Net $\mathrm{CO}_{2}$ uptake per leaf surface area was depressed by low $\mathrm{Zn}$ supply. In addition, the remarkable reduction of total plant leaf area likely affected whole plant photosynthesis and contributed further to the low biomass production under $\mathrm{Zn}$ deficiency conditions. Stomatal conductance was significantly lower in Zn-deficient leaves, which caused in turn lower transpiration. Reduction of stomatal conductance due to low supply of $\mathrm{Zn}$ was reported for other plants such as maize (Wang and Jin 2005) and rice (Hajiboland and Beiramzadeh 2008). Involvement of $\mathrm{Zn}$ in stomatal opening was attributed to the structural role of $\mathrm{Zn}$ in carbonic anhydrase needed for maintaining adequate $\mathrm{HCO}_{3}{ }^{-}$in the guard cells and also to controlling effect of $\mathrm{Zn}$ on $\mathrm{K}^{+}$uptake by the guard cells (Sharma et al., 1995). However, since stomatal aperture is affected also by deficiency of other nutrients such as Fe (Molassiotis et al., 2006 and Hajiboland and Beiramzadeh 2008), it seems to be influenced rather indirectly by factors being common under deficiency of other nutrients, e.g. loss of membrane integrity and passive leakage of $\mathrm{K}^{+}$from guard cells.

The present study aimed to provide the most suitable zinc application in rice to overcome $\mathrm{Zn}$ deficiency problem in rice, by comparing the effects of different methods of $\mathrm{Zn}$ application on rice growth, It was observed that zinc application has significant positive effect on growth, among the varieties, IET24766 recorded significant highest physiological parameters which was followed by IET-25450 and BPT-5204. Zinc application had also significant influence on physiological parameters. The soil base $\mathrm{Zn}$ treatment, $20 \mathrm{~kg}$ $\mathrm{ZnSO}_{4} \mathrm{ha}^{-1}$ at the time of transplanting recorded significantly higher physiological parameters of rice followed by $10 \mathrm{~kg} \mathrm{ZnSO}_{4}$ $\mathrm{ha}^{-1}$ at the time of transplanting and control 00 $\mathrm{kg} \mathrm{ZnSO}_{4} \mathrm{ha}^{-1}$. While, foliar $\mathrm{Zn}$ application recorded significantly maximum physiological parameters of rice under $1 \% \mathrm{Zn}$ EDTA spray 
at tillering and grain filling stage followed by $0 \% \mathrm{Zn}$ EDTA. Further study should be needed to elaborate role of zinc at molecular level for photosynthesis mechanism to strength the knowledge of role of zinc in photosynthetic rate, stomatal conductance and transpiration rate. The obtained results of this study would be beneficial to mitigate $\mathrm{Zn}$ deficiency in rice and therefore, improve zinc use efficiency.

\section{References}

Aravind, P. and Prasad, M. N. V. (2004). Zinc protects chloroplasts and associated photochemical functions in cadmium exposed Ceratophyllum demersum L., a freshwater macrophyte. Plant Science, 166: 1321-1327.

Barak, P., Helmke, P. A. (1993). The chemistry of zinc. In: A. D. Robson Ed. Zinc in soil and plants. Kluwer Academic Publishers, Dordrecht, the Netherlands.

Barber, J. and Anderson, B. (1992). Too much of a good thing: light can be bad for photosynthesis. Trends in Biochemical Sciences, 17: 61-66.

Cakmak, I. (2000). Tansley review number 111: Possible roles of zinc in protecting plant cells from damage by reactive oxygen species. New Phytologist. 146(2): 185-205.

Cakmak, I. (2008). Enrichment of cereal grains with zinc: Agronomic or genetic biofortification. Plant Soil, 302:1-17.

Chen, W. R., Feng, Y. and Chao, Y. E. (2008). Genomic analysis and expression pattern of OsZIP1, OsZIP3 and OsZIP4 in two rice (Oryza sative L.) genotypes with different zinc efficiency. Russian Journal of Plant Physiology, 55:400-409.

Chen, W., He, Z. L., Yang, X. and Feng, Y. (2009). Zinc efficiency is correlated with root morphology, ultrastructure, and antioxidative enzymes in rice. Journal of Plant Nutrition, 32:287305.

Chen, W., Yang, X., He, Z., Feng, Y. and Hu, F. (2008). Differential changes in photosynthetic capacity, 77 K chlorophyll fluorescence and chloroplast ultrastructure between $\mathrm{Zn}$ efficient and $Z n$-inefficient rice genotypes (Oryza sativa) under low zinc stress. Physiology of Plant, 132:89-101.

De Datta, S. K. (1981). Principles and practices of rice production. New York: John Wiley \& Sons.

Dore, V., Koti, R. V. and Hanamaratti, N. G. (2018). Impact of zinc application on morphological and biophysical parameters of rice genotypes in pot experiment. Advances in Research, 16 (2): 1-7.

Fageria, N. K. (2001). Screening method of lowland rice genotypes for zinc uptake efficiency. Scientia Agricola,58:623626.

Fageria, N. K. and Stone, L. F. (2008). Micronutrient deficiency problems in South America. In Micronutrient deficiencies in global crop production, ed. B. J. Alloway, 247-268. New York: Springer.

Fang, W. and Kao, C. H. (2000). Enhanced peroxidase activity in rice leavesin response to excess iron, copper and $\mathrm{Zn}$. Plant Science, 158:71-76.

Hacisalihoglu, G. and Kochian, L. V. (2003). How do some plants tolerate low levels of soil zinc? Mechanisms of zinc efficiency in crop plants. New Phytol., 159: 341-350.

Hacisalihoglu, G., Hart, J. J., Wang, Y., Cakmak, I. and Kochian, L. B. (2003). Zinc efficiency is correlated with enhanced expression and activity of $\mathrm{Cu} / \mathrm{Zn}$ superoxide dismutase and 
carbonic anhydrase in wheat. Plant Physiology, 131:595-602.

Hajiboland, R. and Beiramzadeh, N. (2008). Growth, gas exchange and function of antioxidant defense system in two contrasting rice genotypes under $\mathrm{Zn}$ and Fe deficiency and hypoxia. Acta. Biologica. Szegediensis., 52: 283-294.

Harbinson, J. (1994). The response of thylakoid electron transport and light utilization efficiency to sink limitation of photosynthesis. In: Baker, N. R., and Boyer, J. R. (eds.): Photoinhibition of Photosynthsis. From Molecular Mechanisms to the Field. BIOS Scientific Publishers, Oxford, 273-295.

Hatch, M. D. and Slack, C.R. (1970). Photosynthetic $\mathrm{CO}_{2}$ fixation pathways. Annual Review of Plant Physiology, 21:141-162.

Heyneman, C. A. (1996). Zinc deficiency and taste disorders. Ann. Pharmacother., 30:186-187.

Ignatova, L. K., Rudenko, N. N., Mudrik, V. A., Fedorchuk, T. P. andIvanov, B. N.(2011). Carbonic anhydrase activity in Arabidopsis thaliana thylakoid membrane and fragments enriched with PSI or PSII. Photosynth Res., 110: 89-98.

Jiang, W., Struik, P. C., Keulen, H. V., Zhao, M. and Stomph, T. J. (2008). Does increased $\mathrm{Zn}$ uptake enhance grain $\mathrm{Zn}$ mass concentration in rice? Ann. Appl. Biol., 153: 135-147.

Jyung, W. H., Ehmann, A., Schlender, K. K. and Scala, J. (1975). Zinc nutrition and starch metabolism in Phaseolus vulgaris L. Plant Physiology, 55:414420.

Kabeya, M. J., Shankar, A. G. (2013). Effect of different levels of zinc on growth and uptake ability in rice zinc contrast lines (Oryza sativa L.). Asian Journal of Plant Science and Research, 3(3): 112-116.
Kaul, T., Reddy, P. S., Mahanty, S., Thirulogachandar, V., Reddy, R. A., Kumar, B., Sopory, S. K. and Reddy, M. K. (2011). Biochemical and molecular characterization of stressinduced b-carbonic anhydrase from a C4 plant, Pennisetum glaucum. J. Plant Physiol., 168: 601-610.

Ludwig, M. (2012). Carbonic anhydrase and the molecular evolution ofC4 photosynthesis. Plant Cell Environ., 35: 22-37.

Mandal, B., Hazra, G. C. and Mandal, L. N. (2000). Soil management influences on zinc desorption for rice and maize nutrition. Soil Science Society of America Journal, 64: 1699-1705.

Maret, W. and Sandstead, H. H. (2006). Zinc requirements and the risks and benefits of zinc supplementation. Journal of Trace Element Medical Biology, 20:318.

Maurya, P. K., Yadav, L. M., Thakur, G. and Patel, P. (2018). Effect of micronutrient application growth and yield of Kharif onion (Allium cepa L.). International Journal of Current Microbiology and Applied Sciences, 7(3):601-608.

Molassiotis, A., Tanou, G., Diamantidis, G., Patakas, A. and Therios, I. (2006). Effects of 4-month $\mathrm{Fe}$ deficiency exposure on $\mathrm{Fe}$ reduction mechanism, photosynthetic gas exchange, chlorophyll fluorescence and antioxidant defense in two peach rootstocks differing in Fe deficiency tolerance. Journal of Plant Physiology, 163: 176-185.

Patsikka, E., Kairavuo, M., Sersen, F.,Aro, E. M. and Tyystjarvi, E. (2002). Excess copper predisposes photosystem II to photoinhibition in vivo by outcompeting iron and causing decrease in leaf chlorophyll. Plant Physiology, 129: 1359-1367. 
Prasad, A. S. (1995). Zinc: an overview. Nutrition, 11:93-99.

Prasad, A. S., Beck, F. W., Grabowski, S. M., Kaplan, J. and Mathog, R. H. (1997). Zinc deficiency: changes in cytokine production and T-cell subpopulations in patients with head and neck cancer and in noncancer subjects. Proc. Assoc. Am. Physicians., 109:68-77.

Qadar, A. (2002). Selecting rice genotypes tolerant to zinc deficiency and sodicity stresses, I: Differences in zinc, iron, manganese, copper, phosphorus concentrations, and phosphorus/zinc ratio in their leaves. Journal of Plant Nutrition, 25:457-473.

Qiao, X., He, Y., Wang, Z., Li, X., Zhang, K. and Zeng, H. (2014). Effect of foliar spray of zinc on chloroplast $\beta$-carbonic anhydrase expression and enzyme activity in rice (Oryza sativa L.) leaves. Acta Physiologiae Plantarum, 36: 263-272.

Rehman, H., Aziz, T., Farooq, M., Wakeel, A. and Rengel, Z. (2012). Zinc nutrition in rice production systems, a review. Plant Soil, 361 (1-2): 203-226.

Reynolds, M., Bonnett, D., Chapman, S. C., Furbank, R. T., Mane, Y., Mathe,D. E. and Parry, M. A. (2010). Raising yield potential of wheat. Overview of a consortium approach and breeding strategies. J. Exp. Bot., 62: 439-452.

Riazunnisa, K., Padmavathi, L., Bauwe, H. and Raghavendra, A. S. (2006). Markedly low requirement of added $\mathrm{CO} 2$ for photosynthesis bymesophyll protoplasts of pea (Pisum sativum): possible roles of photorespiratory $\mathrm{CO}_{2}$ and carbonic anhydrase. Physiol Plantarum, 128: 763-772.

Rink, L. and Gabriel, P. (2000). Zinc and the immune system. Proc. Nutr. Soc., 59:541-552.

Saha, S., Chakraborty, M., Padhan, D., Saha, B., Murmu, S., Batabyal, K., Seth, A.,
Hazra, G. C.,Mandal, B. and Bell, R. W. (2017). Agronomic biofortification of zinc in rice: influence of cultivars and zinc application methods on grain yield and zinc bioavailability. Field Crops Research, 210: 52-60.

Samart, S., Chutipaijit, S., Phakamas, N. (2017). Evaluating the effect of zinc oxide nanoparticles on the physiological responses of nine nonphotoperiod sensitive rice cultivars. Materials Today: Proceedings, 4: 6430-6435.

Seilsepour, M. (2006). Study of zinc effects on quantitative and qualitative traits of winter wheat genotypes differing in drought resistance. Crop Production, 2(2): 17-23.

Sharma, P. N., Kumar, N. and Bisht, S. S. (1994). Effect of zinc deficiency on chlorophyll content, photosynthesis and water relations of cauliflower plants. Photosynthetica, 30:353-359.

Sharma, P. N., Tripathi, A. and Bisht, S. S. (1995). Zinc requirement for stomatal opening in cauliflower. Plant Physiology, 107: 751-756.

Shortri, C. K., Tewari, M. N. and Rathore, V. S. (1978). Morphological and ultrastructural abnormalities in zinc deficient maize chloroplasts. Nutritional deficiencies. Plant Biochem. J., 5: 89-96.

Sillanpaa, M. (1982). Micronutrients and nutrient status of soils: A global study(Soils Bulletin 48). Rome, Italy: FAO.

Simmer, K., Thompson, R. P. (1985). Zinc in the fetus and newborn. Acta. Paediatr. Scand. Suppl., 319:158-163.

So, A. K., Espie, G. S. (2005). Cyanobacterial carbonic anhydrases. Can JBot., 83: 721-734.

Solomons, N. W. (1998). Mild human zinc deficiency produces an imbalance 
between cell-mediated and humoral immunity. Nutrition Review, 56:27-28.

Srivastava, P. C. and Gupta, U. C. (1996). Trace elements in crop production. Science Publishers, Lebanon.

Storey, J. B. (2007). Zinc. In: Barker, A. V., Pilbeam, D. J. (eds) Handbook of plant nutrition. CRC Press, Taylor \& Francis Group, Boca Raton, pp 411-436.

Tems, U., Burnell, J. N. (2010). Characterization and expression of the maize-carbonic anhydrase gene repeat regions. Plant PhysiolBioch., 48: 945951.
Thomson, W. W. and Weiser, T. E. (1962). The fine structure of chloroplasts from mineral deficient leaves of Phaseolus vulgaris. Am. J. Bot., 49: 1047.

Tobin, A. J. (1970). Carbonic anhydrase from parsley leaves. J. Biol. Chem., 245: 2656-3666.

Wang, H. and Jin, J. Y. (2005). Photosyntheric rate, chlorophyll fluorescence parameters, and lipid peroxidation of maize leaves as affected by zinc deficiency. Photosynthetica, 43: 591596.

\section{How to cite this article:}

Vivek N. Zinzala, Ajay V. Narwade, Nilima Karmakar and Patel, P.B. 2019. Influence of Zinc Applications on Photosynthesis, Transpiration and Stomatal Conductance in Kharif Rice (Oryza sativa L.) Genotypes. Int.J.Curr.Microbiol.App.Sci. 8(10): 150-168. doi: https://doi.org/10.20546/ijcmas.2019.810.016 